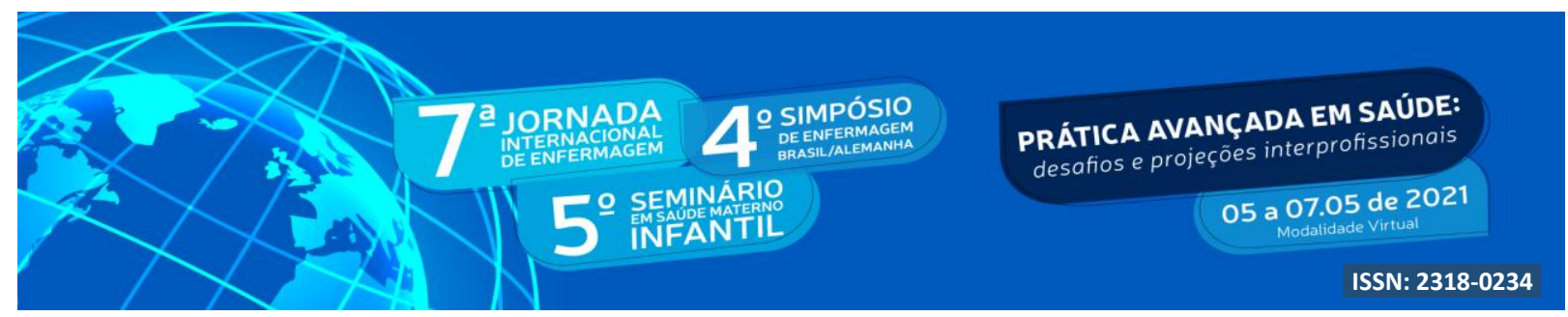

DOI: http://doi.org/10.48195/jie2021-056

\title{
RODA DE CONVERSA ONLINE SOBRE ARTETERAPIA E MUSICOTERAPIA EM TEMPOS DE PANDEMIA ${ }^{1}$
}

\author{
Flávia Camef Dorneles Lenz ${ }^{2}$; Nathália Fortes Schlotfeldt ${ }^{3}$; Claudete Moreschi ${ }^{4}$. \\ RESUMO
}

Este trabalho tem com objetivo relatar a experiência acadêmica de bolsistas voluntárias acerca da atuação em roda de conversa online que abordou sobre arteterapia e musicoterapia durante a pandemia COVID-19. Trata-se de uma experiência desenvolvida no Laboratório de Práticas Integrativas e Complementares em Saúde de uma universidade da região centro oeste do Rio Grande do Sul. A prática foi realizada por meio da plataforma Google Meet. Foi possível perceber, por meio deste estudo, que a não presencialidade não interferiu de forma negativa na realização das práticas, uma vez que os recursos tecnológicos possibilitaram aos participantes receberem a atividade de forma remota.

Palavras-chave: Enfermagem; Práticas Integrativas e Complementares; Promoção da saúde.

\begin{abstract}
This paper aims to report on the academic experience of voluntary fellows on the performance of an online conversation circle that addressed art therapy and music therapy during the COVID-19 pandemic. It is an experience developed at the Laboratory of Integrative and Complementary Practices in Health of a university in the central west region of Rio Grande do Sul. The practice was carried out through the Google Meet platform. It was possible to perceive, through this study, that the lack of presence did not interfere in a negative way in the realization of the practices, since the technological resources enabled the participants to receive the activity remotely.
\end{abstract}

Key Words: Nursing; Integrative and Complementary Practices; Health promotion.

\section{INTRODUÇÃO}

\footnotetext{
${ }^{1}$ Relato de experiência

${ }^{2}$ Estudante do Curso de Enfermagem. Universidade Regional Integrada do Alto Uruguai e das Missões- Campus Santiago. E-mail: flaviacamefd@ gmail.com

${ }^{3}$ Estudante do Curso de Enfermagem. Universidade Regional Integrada do Alto Uruguai e das Missões- Campus Santiago. E-mail: nathaliafschlotdelft@gmail.com

${ }^{3}$ Orientador. Enfermeira. Doutora em Ciências: Ambiente e Desenvolvimento. Universidade Regional Integrada do Alto Uruguai e das Missões- Campus Santiago. E-mail:clau_moreschi@yahoo.com.br
} 


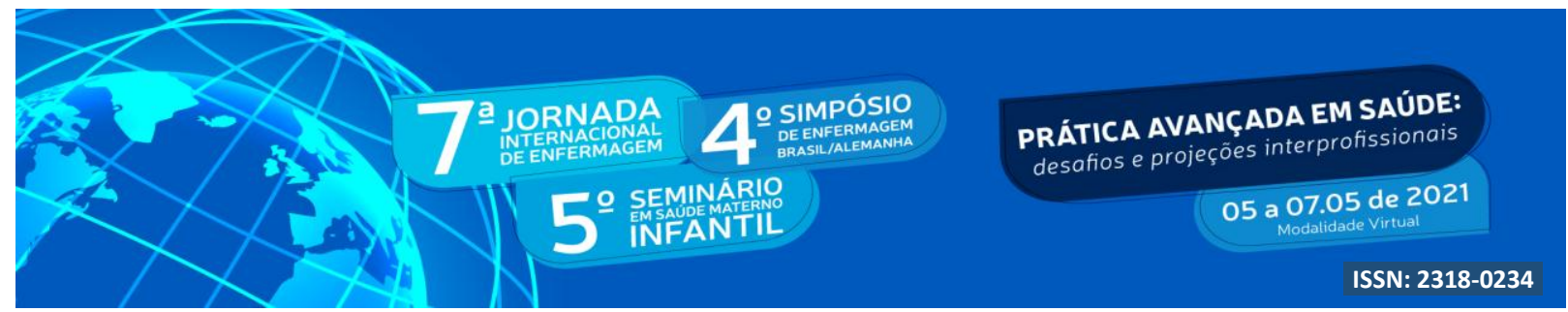

Ações de promoção da saúde ganharam visibilidade enquanto estratégia para enfrentar os diversos problemas de saúde que assolam as populações humanas e o seu entorno. A promoção de saúde consiste na realização de atividades de transformação de comportamento dos usuários, estando relacionada a todas as práticas que possam vir a ofertar uma melhoria na qualidade de vida da população, não se limitando apenas a solução de problemas já instalados, mas sim visando aumentar a saúde e o bem-estar como um todo (GUERIN et al., 2017).

A partir da década de 70 outros determinantes da saúde (biológicos, ambientais e de estilo de vida) tornaram-se mais relevantes, levando a dúvidas sobre à eficácia da biomedicina como único modelo de atenção à saúde pública. Nesse sentido, tendo em vista ainda, o alto custo das ações biomédicas, emerge um notório interesse da sociedade ocidental pelas práticas terapêuticas que não pertencentes à medicina científica (HABIMORAD et al., 2020)

As Práticas Integrativas e Complementares em Saúde (PICS) são práticas que buscam estimular os mecanismos naturais de prevenção de agravos e recuperação da saúde por meio de ações efetivas. Por meio delas são empregados recursos naturais no cuidado à saúde, substituindo o uso de substâncias que não existam na natureza, fugindo do modelo biomédico e da medicalização (MENDES, et al., 2019).

No Brasil, as PICS ganham força no ano de 2006 onde por meio da portaria $\mathrm{n}^{\mathrm{o}}$ 971/2006, o Ministério da Saúde (MS) publica a Política Nacional de Práticas Integrativas e Complementares em Saúde (PNPIC). Sendo vinculada ao Sistema Único de Saúde (SUS), ela vem com o objetivo de garantir a integralidade dos serviços de saúde. A partir disso, diversas práticas tais como, fitoterapia, acunpuntura, e homeopatia, entre outras, passaram a fazer parte das ofertas de cuidado do SUS (RUELA, et al., 2019).

No ano de 2017, a portaria $n^{\circ} 849 / 2017$ de 27 de março, trouxe consigo um grande avanço no uso das PICS. Por meio dela, foram incluídas mais 14 PICS para compor o quadro de oferta no SUS, sendo elas: Arteterapia, Ayurveda, Biodança, Dança Circular, Meditação, Musicoterapia, Naturopatia, Osteopatia, Quiropraxia, Reflexoterapia, Reiki, Shantala, Terapia Comunitária Integrativa e Yoga (MENDES, et al., 2019).

Além disso, cabe ressaltar, que a implantação da PNPIC teve caráter político, técnico, 


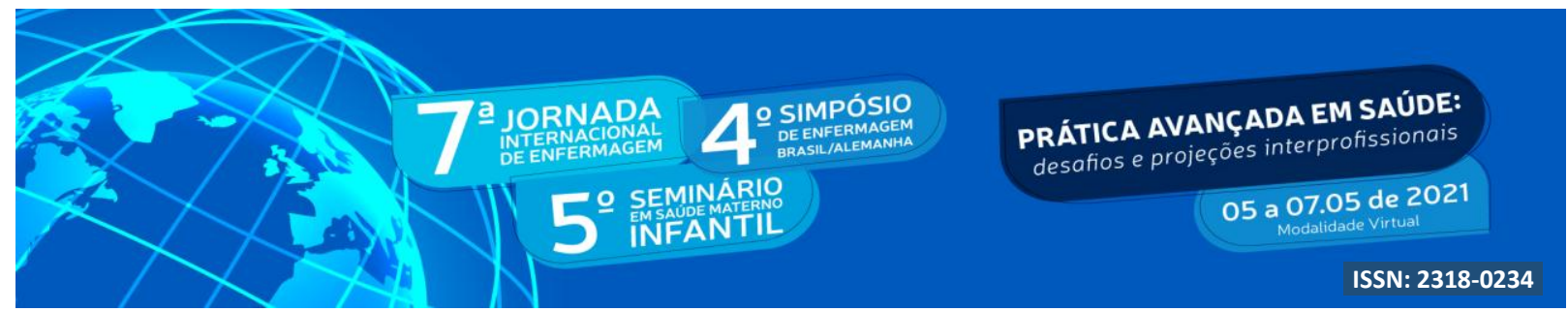

econômico, social e cultural, uma vez que estabeleceu diretrizes nacionais para o uso das PIC, a partir de experiências e práticas já adotadas nos serviços de saúde que obtiveram resultados satisfatórios. Tal fato possibilitou ainda mais a difusão dessas práticas em diversos pontos do país (RUELA, et al., 2019).

\section{OBJETIVO}

Este trabalho tem como objetivo relatar a experiência acadêmica de bolsistas voluntárias acerca da atuação em roda de conversa online que abordou sobre arteterapia e musicoterapia durante a pandemia COVID-19.

\section{METODOLOGIA}

Trata-se de um relato de experiência vivenciado por acadêmicas de enfermagem, enquanto bolsistas de um projeto de Ação Social intitulado: Laboratório de Práticas Integrativas e Complementares em Saúde - LAPICS, em uma universidade do centro oeste do Rio Grande do Sul.

As atividades ocorreram de forma remota via plataforma Google Meet, devido às necessidades impostas pela pandemia mundial COVID- 19. Ocorreram cinco encontros ao longo do semestre letivo, com diferentes temáticas e participação de diversos profissionais, comunidade acadêmica e comunidade em geral.

Neste trabalho será relatada a primeira Roda de Conversa Online, que abordou a temática Arteterapia e Musicoterapia.

\section{RESULTADOS E DISCUSSÃO}

A musicoterapia é definida como um instrumento que se utiliza experiências musicais para auxiliar na promoção da saúde do cliente. O uso desta terapia melhora a relação de comunicação, organização, aprendizagem expressão e mobilização, atingindo um melhor efeito terapêutico (MENDES et al., 2015).

Já a arteterapia é uma prática que utiliza a arte como base do processo terapêutico. É composta por diferentes técnicas expressivas como pintura, desenho, sons, música, 


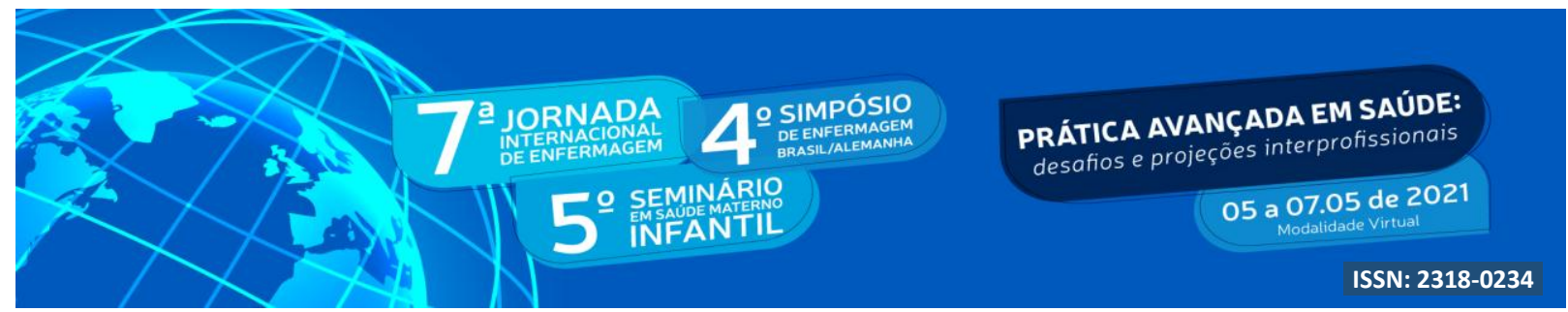

modelagem, colagem, mímica, tecelagem, expressão corporal, escultura, dentre outras. Pode ser realizada em grupo ou individualmente. Além disso, estimula a expressão criativa, favorecendo o desenvolvimento motor, o raciocínio e o relacionamento afetivo (BRASIL, 2017).

Este encontro foi o primeiro a ser realizado e ocorreu dia 16 de setembro de 2020, com duração de uma hora, com a fala de uma profissional formada na área. Durante a roda de conversa foi possível refletir acerca dos benefícios da aplicação dessas PICS, bem como repensar sobre formas alternativas de promover qualidade de vida aos pacientes atendidos.

Dentre as diversas vantagens das PICS destacam-se, os tratamentos não farmacológicos, com baixas contraindicações, de menor custo, que proporcionam a sensação de bem-estar geral no corpo e para a mente e podem ser usadas por qualquer indivíduo. Contribuem ainda, para equilibrar os gastos no sistema, favorecem a prevenção, estimulam a reabilitação mais rápida e com menos efeitos colaterais, além da redução do consumo de medicamentos, de outros procedimentos e internações (BEZERRA et al., 2020).

Por meio deste encontro foi possível estabelecer um diálogo com compartilhamento de experiências frente à temática, e ainda, vale reassaltar que, de forma online, os participantes puderam usufruir da aplicação da Musicoterapia e desfrutar de um momento relaxante, reflexivo e de conexão consigo mesmo.

Mediante divulgação, neste encontrou participaram pessoas de diferentes cidades do Rio Grande do Sul, bem como de outros Estados. Dentre estas, estavam, acadêmicos dos cursos de Enfermagem, Farmácia, Terapia Ocupacional e Pedagogia, mestrandos, bem como profissionais da Enfermagem, Medicina e Arteterapeutas, entre outros, totalizando 55 pessoas alcançadas nesta roda de conversa online.

A integração de diversos públicos, dos mais diferentes locais, levando conhecimento por meio de formas alternativas, foi possível, preservando a qualidade da realização das atividades, e buscando a reinvenção diante daquilo que o cenário proporciou. Tornando notório que a inovação na área da saúde é um campo em expansão, com possibilidades muito favoráveis, estimulando o pensar sobre alguns aspectos inerentes que são indispensáveis na interface com o debate tecnológico, pois as tecnologias atuais e futuras podem ser aplicadas no dia a dia em diferentes campos de assistência ao paciente (BARROS, et al., 2021) 


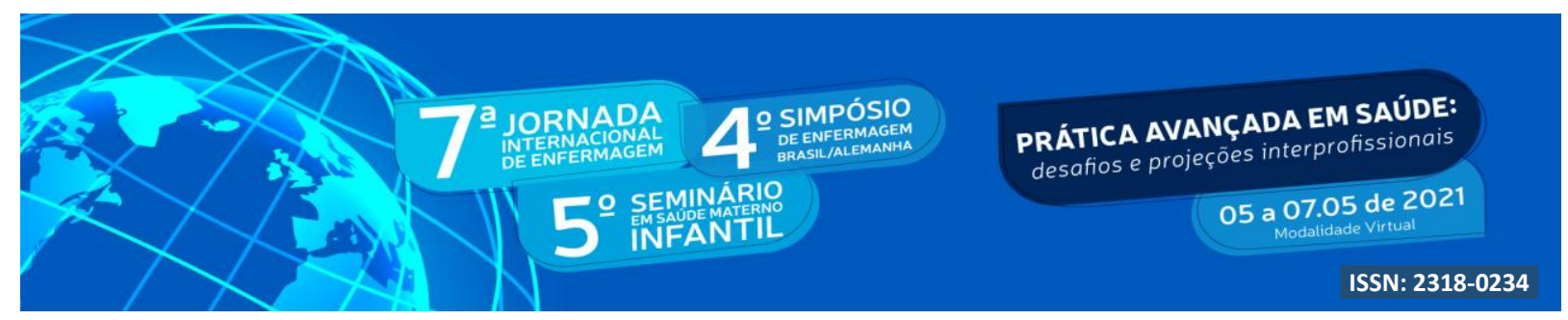

\section{CONCLUSÃO}

Por meio desta atividade pode-se perceber o quão importante é a integração profissionais, comunidade acadêmica e comunidade em geral, e o quanto tecnologia contribui para essa interação. Uma vez que, aproxima pessoas diferentes, de diversos lugares, com distintas necessidades e diferenciadas formações e ainda assim, promove maneiras de facilitar a promoção da saúde de forma integral.

No que diz respeito as PICS, foi possível perceber, por meio deste estudo, que a não presencialidade não interferiu de forma negativa na realização das práticas, uma vez que os recursos tecnológicos possibilitaram os participantes de receberem a atividade de forma remota.

Portanto cabe destacar que, diante do cenário atual, mais do que nunca, exige-se dos profissionais a busca por atualizações no que se refere ao uso de tecnlogias em saúde. No que tange a enfermagem, é indispensável pensar e refletir acerca da necessidade de reinvenções e adaptações, que permitam a continuidade do cuidado de forma a favorecer a promoção da saúde mesmo diante de todos os desafios impostos.

\section{REFERÊNCIAS}

BARROS, E N. L. ET AL. O uso das tecnologias auxiliadoras à saúde: desafios e benefícios. Diversitas Journal, v. 6, n.1, pp: 698-712, 2021.

BEZERRA, D. R. C. ET AL. Uso de Práticas Integrativas e Complementares no período de isolamento social da COVID- 19 no Brasil. Research, Society and Development, v. 9, n. 11, e1329119718, 2020.

BRASIL. Ministério da Saúde. Portaria no 849 de 27 de março de 2017. Brasil, 2017.

GUERIN, C. S. ET AL. Promovendo a educação em saúde no espaço não formal de aprendizagem. Rev Brasileira de Promoção da Saúde, v.30, n. 1, pp: 5-12, 2017.

HABIMORAD, P. H. L. ET AL. Potencialidades e fragilidades da implementação da Política 


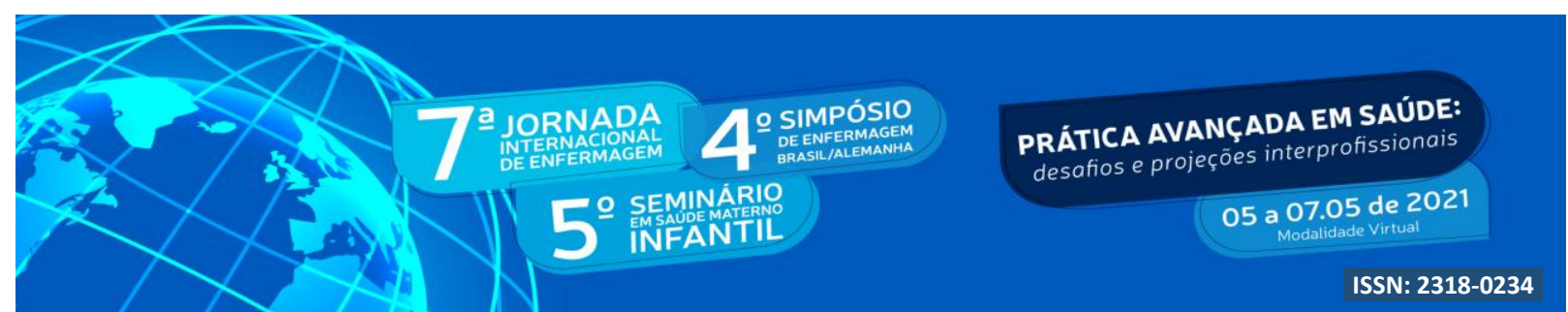

Nacional de Práticas Integrativas e Complementares. Ciência \& Saúde Coletiva, v. 25, n. 2, pp: 395-405, 2020.

MENDES, D. S. et al. Benefícios das práticas integrativas e complementares no cuidado de enfermagem. Journal Health NPEPS. v. 4, n.1, p.:302-318, 2019.

MENDES, M. V. dos S. et al. Crianças com retardo do desenvolvimento neuropsicomotor: musicoterapia promovendo qualidade de vida. Rev. Bras. Enferm. vol.68 no.5 Brasília Sept./Oct. 2015.

RUELA, L. O. et al. Implementação, acesso e uso das práticas integrativas e complementares no Sistema Único de Saúde: revisão da literatura. Ciênc. saúde coletiva, v. 24, n.11, 2019. 